\title{
Thermal Properties, Interactions, Morphologies, and Conductivity Behavior in Blends of Poly(vinylpyridine)s and Zinc Perchlorate
}

\author{
Shiao-Wei Kuo, Chung-Hsi Wu, and Feng-Chih Chang* \\ Institute of Applied Chemistry, National Chiao-Tung University, Hsin-Chu, Taiwan \\ Received November 4, 2003
}

\begin{abstract}
We have investigated the physical properties and specific interaction of two regioisomeric poly(vinylpyridine)s-poly(2-vinylpyridine) (P2VP) and poly(4-vinylpyridine) (P4VP)-blended with zinc perchlorate. Analyses by differential scanning calorimetry indicate that the values of $T_{g}$ of the poly(vinylpyridine)s increase significantly upon blending with zinc perchlorate. The values of $\mathrm{T}_{\mathrm{g}}$ of P2VP and P4VP increase by 119 and $154^{\circ} \mathrm{C}$, respectively, relative to their corresponding pure polymers, upon blending with 40 and 30 wt \%, respectively, zinc perchlorate. On the basis of the extended configuration entropy model equation, the interaction strength of the zinc salt/P4VP blend (3.60) is greater than that of the zinc salt/P2VP blend (3.55). The interaction between the polymer and the zinc ion was confirmed by infrared, X-ray photoelectron, and solid-state NMR spectroscopies, which suggest that the zinc cation coordinates to pyridine units in a metal-ligand bonding mode. In addition, the strength of the interaction between the polymer and the zinc ion is influenced by the different steric demands of the two polymer structures. A good correlation is found in the morphol ogy of these polymer/zinc salt blends as determined by wide-angle X-ray diffraction and solid-state $\mathrm{NMR} \mathrm{T}_{1_{\rho}}{ }^{\mathrm{H}}$ measurements.
\end{abstract}

\section{Introduction}

The physical properties of a polymer can be modified to create novel materials by blending it with another component with which it can undergo specific noncovalent interactions, such as hydrogen-bonding or ionic interactions. Blending polymers with inorganic salts is quite useful for producing new materials having enhanced thermal, mechanical, and separation (e.g., of ol efin/paraffin gas mixtures) properties. ${ }^{1-4}$ In a previous study, ${ }^{5}$ we described the effects of hydrogen-bonding interactions on the thermal properties and miscibility behaviors of poly(vinylpyridine)s blended with phenolic resin. In this paper, we extend this concept by investigating how noncovalent bonding can affect the thermal and conductive properties of polymers by blending zinc perchlorate with poly(vinylpyridine)s.

A study of the thermal behavior and ionic interactions of poly(2-vinylpyridine) (P2VP) and poly(4-vinylpyridine) (P4VP), used as conducting polymers with copper iodide as the conducting medium, has been reported. ${ }^{6}$ Furthermore, many reports have appeared describing ionomers formed using P2VP and P4VP. ${ }^{7-14}$ The zinc perchlorate employed in this study was coordinated to either P2VP or P4VP by blending in solution. According to the hard/soft and acid/base concepts, ${ }^{15}$ the water of hydration can be considered a hard base, the zinc cation a borderline acid, and the pyridine ligand a borderline base. The metal-ligand complex is formed by displacing the water of hydration by the pyridine ligand. According to the Bronsted acid ionization constants ${ }^{14}$ and the Guttman donor-acceptor numbers, ${ }^{15}$ the pyridine ligand is a stronger base than is water.

The specific interaction generally can be characterized by Fourier transform infrared spectroscopy (FTIR) because this interaction affects the local electron densities and correspondingly frequency shifts can be

* To whom correspondence should be addressed. E-mail: changfc@cc.nctu.edu.tw. Phone: 886-3-5727077. Fax: 886-35719507. observed. ${ }^{16-18}$ Recently, X-ray photoel ectron spectroscopy (XPS) has also been used to study the specific interaction between the metal and ligand in polymer blends. ${ }^{19-21}$ The devel opment of a new peak or shoulder usually can be observed in the XPS spectrum when the chemical environment of an atom in a polymer blend is perturbed as a result of a specific interaction. In addition to IR and XPS spectroscopies, solid-state NMR spectroscopy also provides a powerful tool for studying the specific interactions and the resulting molecular mobility of a polymer involved in such an interaction formation. ${ }^{22-26}$ The ${ }^{13} \mathrm{C}$ NMR spectroscopic chemical shifts and line shapes observed in cross-polarization and magic-angle spinning (CP/MAS) spectra can identify the chemical environments of carbon atoms in the blend since these features are highly sensitive to the local electron density. Moreover, the phase behavior and the morphology of polymer blends can be estimated from the proton spin-lattice relaxation time in the rotating frame $\left(\mathrm{T}_{1 \rho}{ }^{\mathrm{H}}\right)$ measured by solid-state N MR spectroscopy. The purposes of this study were to investigate the thermal properties, interactions, morphologies, and conductivity behavior found in blends of zinc perchlorate and two isomeric poly(vinylpyridine)s.

\section{Experimental Section}

Materials. P2VP having $M_{n}=40000$ was obtained from Scientific Polymer Products. P4VP $\left(M_{w}=60000\right)$ was obtained from Aldrich. Zinc perchlorate hexahydrate $\left(\mathrm{Zn}\left(\mathrm{ClO}_{4}\right)_{2} \cdot 6 \mathrm{H}_{2} \mathrm{O}\right)$, which was also obtained from Aldrich, was dried in a vacuum oven at $70^{\circ} \mathrm{C}$ for $24 \mathrm{~h}$. N,N-Dimethylformamide (DMF) was used as received.

Preparation of Samples of Polymer/Salt Mixtures. Zinc salt/P2VP and zinc salt/P4VP blends having varying zinc contents were prepared by dissolving the desired amounts of the respective polymer and $\mathrm{Zn}\left(\mathrm{ClO}_{4}\right)_{2} \cdot 6 \mathrm{H}_{2} \mathrm{O}$ in DMF. After continuous ultrasonic stirring for $12 \mathrm{~h}$, the solutions were maintained at $50{ }^{\circ} \mathrm{C}$ for $24 \mathrm{~h}$ on Teflon plates to facilitate desolvation, and then dried further under vacuum at $90{ }^{\circ} \mathrm{C}$ for $2 \mathrm{~d}$.

Characterizations. The measurement of thermal properties was conducted on a TA Instrument DSC 2010. The 


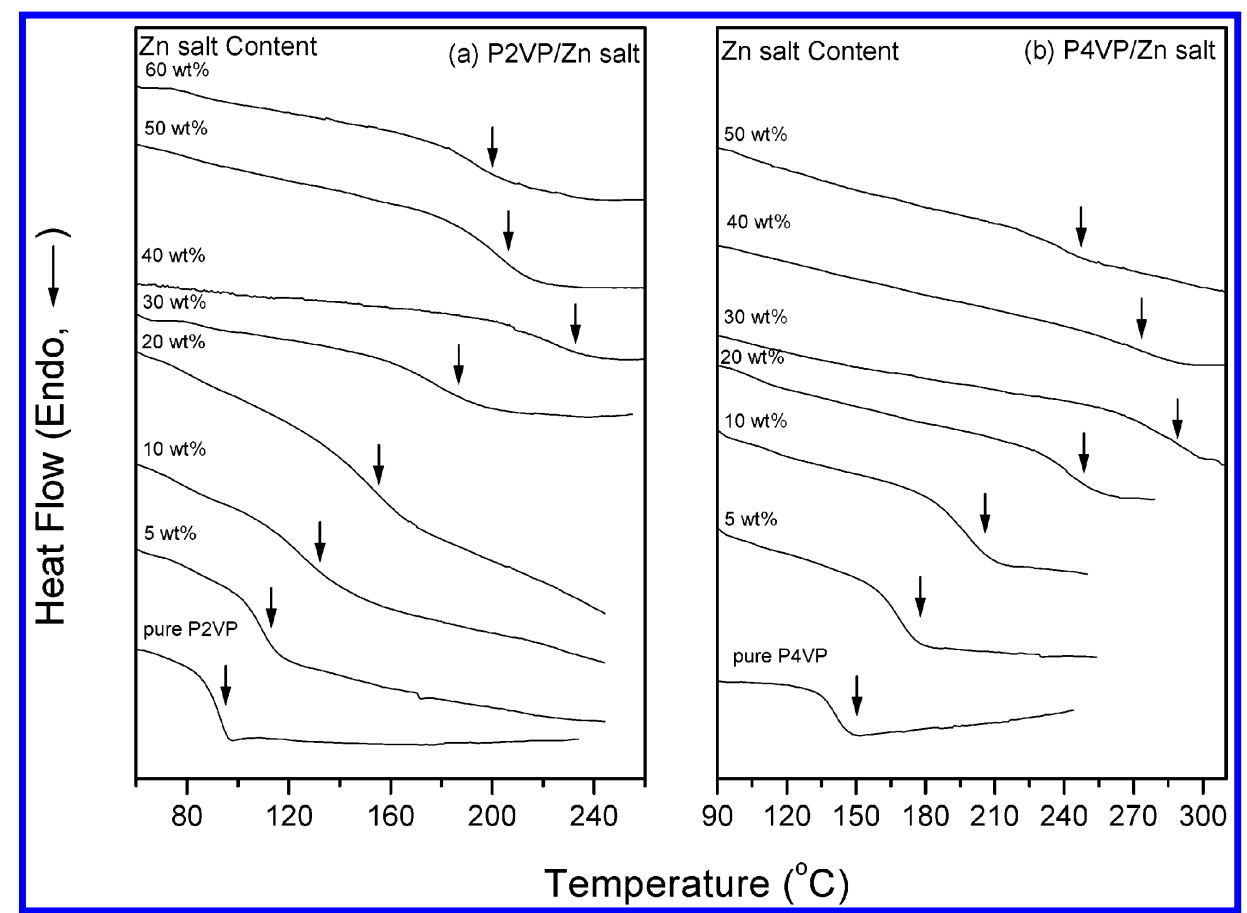

Figure 1. DSC scans for (a) zinc salt/P2VP and (b) zinc salt/P4VP blends having varying compositions.

measurement was conducted under a nitrogen flow rate of ca. $25 \mathrm{~mL} / \mathrm{min}$. All samples were sequentially heated to $300{ }^{\circ} \mathrm{C}$, annealed for $3 \mathrm{~min}$, cooled rapidly to $20^{\circ} \mathrm{C}$, and then reheated. A heating rate of $20^{\circ} \mathrm{C} / \mathrm{min}$ was applied in all cases. The glass transition temperature $\left(T_{g}\right)$ was taken as the midpoint of this glass transition.

I onic conductivity measurements were conducted on an Autolab (Eco Chemie) with alternating current in the frequency range from $10 \mathrm{~Hz}$ to $10 \mathrm{MHz}$. A blend film dried at $115{ }^{\circ} \mathrm{C}$ for $24 \mathrm{~h}$ was sandwiched between stainless steel blocking el ectrodes ( $1 \mathrm{~cm}$ diameter). The specimen thickness was ca. $110 \mu \mathrm{m}$, and the impedance response was measured under ambient temperature.

All infrared spectra were recorded in the range of 4000$400 \mathrm{~cm}^{-1}$ with spectral resolution of $1 \mathrm{~cm}^{-1}$, over 32 scans on a Nicolet Avatar 320 FT-IR spectrophotometer. Infrared spectra of blend films were measured using the conventional $\mathrm{KBr}$ disk method. A DMF solution of the mixture was cast onto a $\mathrm{KBr}$ disk, and the solvent was evaporated at $90{ }^{\circ} \mathrm{C}$ for $24 \mathrm{~h}$. IR spectra were obtained at $120^{\circ} \mathrm{C}$ by mounting the cell inside the temperature-controlled compartment of the spectrometer. The films used in this study were sufficiently thin to obey the Beer-Lambert law. High-resolution solid-state NMR spectroscopy experiments were performed at room temperature using a Bruker DSX-400 spectrometer operating at resonance frequencies of 399.53 and $100.47 \mathrm{M} \mathrm{Hz}$ for $^{1} \mathrm{H}$ and ${ }^{13} \mathrm{C}$ nuclei, respectively. The ${ }^{13} \mathrm{C} \mathrm{CP} / \mathrm{MAS}$ NMR spectra were measured with a $3.9 \mu \mathrm{S} 90^{\circ}$ pulse, a $3 \mathrm{~s}$ pulse delay time, an acquisition time of $30 \mathrm{~ms}$, and 2048 scans. All NMR spectra were taken at $300 \mathrm{~K}$ using broad-band proton decoupling and a normal cross-polarization pulse sequence. A magic-angle sample-spinning (MAS) rate of $5.4 \mathrm{kHz}$ was used to avoid absorption overlapping. The proton spin-lattice relaxation time in the rotating frame $\left(\mathrm{T}_{1 \rho}{ }^{\mathrm{H}}\right)$ was determined indirectly by carbon nucleus observation using a $90^{\circ}-\tau$-spin lock pulse sequence prior to cross-pol arization. The data acquisition was performed by ${ }^{1} \mathrm{H}$ decoupling with delay time $(\tau)$ ranging from 0.1 to $12 \mathrm{~ms}$ and a contact time of $1.0 \mathrm{~ms}$.

To observe the crystalline structures of the zinc salt or the polymer matrix, X-ray diffraction spectra were collected on an M 18XHF-SRA instrument (MacScience Co., J apan) using Cu $K \alpha$ radiation with an X-ray wavelength $(\lambda)$ of $1.5405 \AA$ under $50 \mathrm{kV}$ and $200 \mathrm{~mA}$ in the range of $2 \theta=5-50$.

\section{Results and Discussion}

Analysis of Thermal Properties. According to the results of a previous study, ${ }^{27}$ the properties of a polymer/ salt mixture can be changed dramatically as a result of ionic aggregation. First of all, we carried out thermal analyses to determine whether the properties of these systems are affected by the addition of the zinc salt. Figure 1 shows DSC analyses of the zinc salt/P2VP and zinc salt/P4VP blends of various compositions. The values of $\mathrm{T}_{\mathrm{g}}$ of pure P2VP and pure P4VP are 90 and $144{ }^{\circ} \mathrm{C}$, respectively. Figure 2 shows that the maximum enhancement of the $\mathrm{T}_{\mathrm{g}}$ is ca. $119^{\circ} \mathrm{C}$ for P2VP containing 40 wt $\%$ zinc salt and ca. $154{ }^{\circ} \mathrm{C}$ for P4VP in the presence of 30 wt \% zinc salt. Recently, Kim et al. proposed an extended configuration entropy model to predict the $\mathrm{T}_{\mathrm{g}}$ behavior of polymer/salt complex systems. ${ }^{28}$ This equation is

$$
\begin{aligned}
& \ln \frac{\mathrm{T}_{\mathrm{g}_{12}}}{\mathrm{~T}_{\mathrm{g}_{1}}}=\beta\left[\left(1-\gamma_{12} \ln \left(\frac{\mathrm{z}-1}{\mathrm{e}}\right)\right)\left(\frac{\phi_{1}}{\mathrm{r}_{1}} \ln \phi_{1}+\frac{\phi_{2}}{\mathrm{r}_{2}} \ln \phi_{2}\right)-\right. \\
& \left.\frac{\phi_{2}}{r_{2} \mathrm{vm}} \frac{4}{3} A_{\text {diss }} \mathrm{I}_{3 / 2} \tau\left(I^{1 / 2}\right)\right]
\end{aligned}
$$

where $T_{g_{1}}$ and $T_{g_{12}}$ are the glass transition temperatures of the pure polymer and the polymer/salt blend, respectively. The terms $\phi_{i}$ and $r_{i}$ are the volume fraction and the degree of polymerization for component $\mathrm{i}$, respectively; $r_{2}=1$. The term $\beta=z R / M_{1 u} \Delta C_{p p}$, where $z$ is the lattice coordination number, $R$ is the gas constant, $M_{1 u}$ is the molecular weight of the repeating unit of the polymer, and $\Delta C_{p p}$ is the isobaric specific heat of the polymer. The term $r_{12}$ is a proportionality constant representing the strength of the interaction between the polymer and the salt. The term $\left(\phi_{2} / \mathrm{r}_{2} \mathrm{Vm}\right)(4 / 3) \mathrm{A}_{\text {diss }} \mathrm{I}_{3 / 2} \tau$ $\left(I^{1 / 2}\right)=A^{D H}$ accounts for the cation-anion interaction. Table 1 lists the molecular weights, values of $T_{g}$, specific heats, and densities of components used in this study. The ionic interactions or ionic cluster formation in the amorphous regime of the ionomers usually resembles 


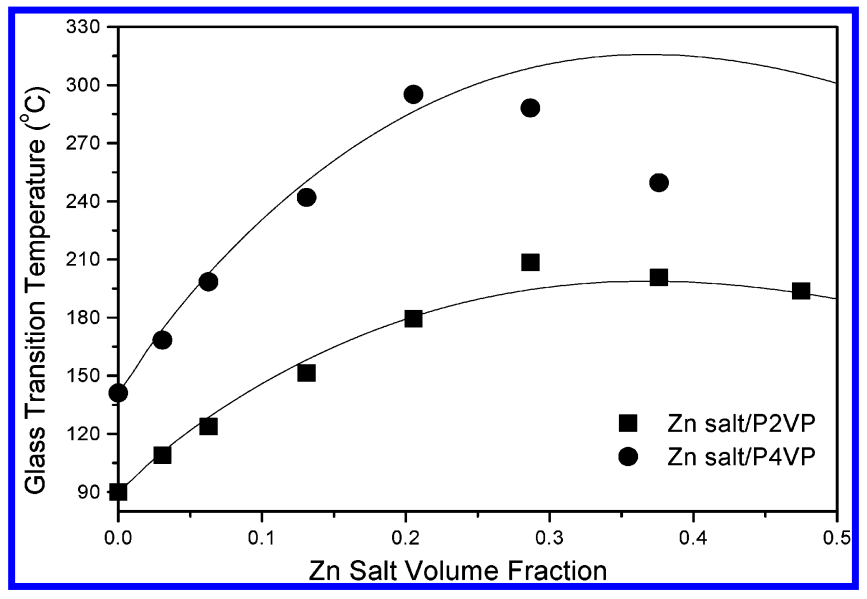

Figure 2. Variation in the value of $T_{g}$ of $P 2 V P$ and $P 4 V P$ blends with zinc perchlorate. The solid lines are calculated from the configuration entropy model.

Table 1. List of Molecular Weights, Values of $\mathbf{T}_{\mathbf{g}}$, Specific Heats, and Densities of the Components Used in This Study

\begin{tabular}{|c|c|c|c|c|}
\hline & $\mathrm{M}_{\mathrm{w}}$ & $\mathrm{T}_{\mathrm{g}},{ }^{\circ} \mathrm{C}$ & $\begin{array}{c}\Delta \mathrm{C}_{\mathrm{pp}}{ }^{\mathrm{a}} \\
\mathrm{J} /(\mathrm{kg} \mathrm{K})\end{array}$ & $\begin{array}{l}\text { density, } \\
\mathrm{g} / \mathrm{cm}^{3}\end{array}$ \\
\hline $\begin{array}{l}\text { P2VP } \\
\text { P4VP } \\
\mathrm{Zn}(\mathrm{ClO} 4)_{2} \cdot 6 \mathrm{H}_{2} \mathrm{O}\end{array}$ & $\begin{array}{r}40000 \\
60000 \\
372\end{array}$ & $\begin{array}{r}90 \\
141\end{array}$ & $\begin{array}{l}1680 \\
1680\end{array}$ & $\begin{array}{l}1.257^{a} \\
1.257^{a} \\
2.052^{b}\end{array}$ \\
\hline blend system & $Z$ & $\beta$ & $r_{12}$ & $A^{D H}$ \\
\hline $\begin{array}{l}\mathrm{P} 2 \mathrm{VP} / \mathrm{Zn}(\mathrm{ClO} 4)_{2} \\
\mathrm{P} 4 \mathrm{VP} / \mathrm{Zn}(\mathrm{ClO} 4)_{2}\end{array}$ & $\begin{array}{l}12 \\
12\end{array}$ & $\begin{array}{l}0.565 \\
0.565\end{array}$ & $\begin{array}{l}3.55 \\
3.60\end{array}$ & $\begin{array}{l}0.06 \\
0.06\end{array}$ \\
\hline
\end{tabular}

a Values obtained from the group contribution method. ${ }^{42}$ b Value obtained from the density measurement. ${ }^{17}$

physical cross-linking. The mobility of polymer chains is restricted by such physical cross-linking and, thus, leads to higher glass transition temperatures relative to that of the mother polymer. Generally, the value of $\mathrm{T}_{\mathrm{g}}$ increases gradually upon the addition of the salt because of increased ion-polymer and ion-ion interactions, and a maximum glass transition temperature is achieved at a certain content of the zinc salt; an excessive amount of the zinc salt tends to decrease the value of $T_{g}$ because of increasing interchain distance above the optimized zinc salt content. ${ }^{29}$ Figure 2 presents the variation in the value of $T_{g}$ as a function of the zinc salt content; the solid lines were calculated from eq 1 and show that a good correlation exists between the experimental data and the model's predictions for both P2VP and P4VP systems at lower zinc salt content. The larger deviation was found for the P4VP system at higher zinc salt content, but in this case, the value of $\mathrm{A}^{\mathrm{DH}}$ should be constant between those of the P2VP and P4VP systems because of the same zinc salt. However, only taking into account the interaction parameter strength, the interaction parameter for the zinc salt/ P4VP blend $\left(r_{12}=3.60, A^{D H}=0.06\right)$ is greater than that of the zinc salt/P2VP blend $\left(r_{12}=3.55, A^{D H}=0.06\right)$, indicating that the stronger interactions are those between the zinc salt and P4VP. In addition, a higher zinc salt content is required for P2VP to achieve its maximum enhancement in the value of $T_{g}$, which results presumably from the relatively hindered position of the nitrogen atoms' lone pairs of electrons that neighbor the polymer main chain. The zinc cation interacts less effectively with these hindered nitrogen atoms in P2VP, and thus, a higher zinc ion content is required to achieve

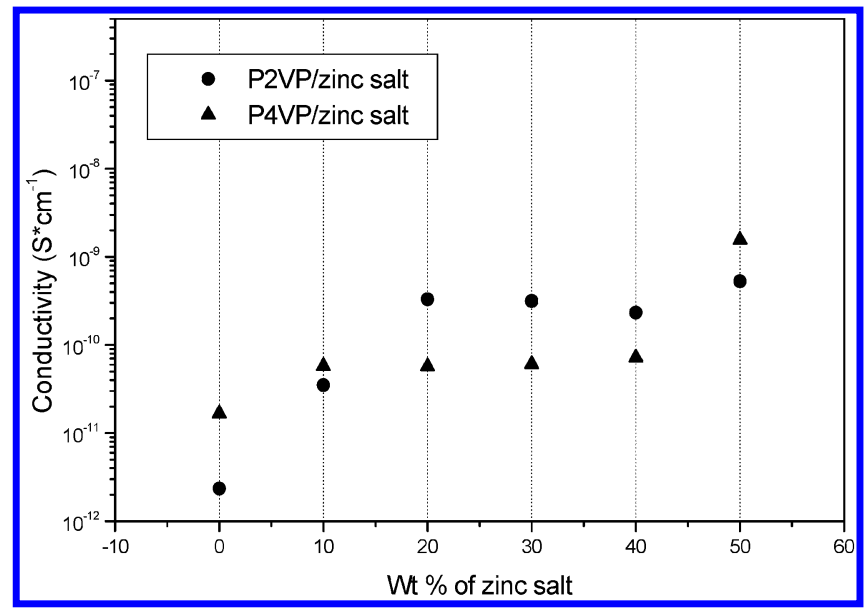

Figure 3. Conductivity behavior of P2VP and P4VP blended with zinc perchlorate as a function of the zinc concentration.

the maximum cross-linking and, hence, the maximum value of $\mathrm{T}_{\mathrm{g}}$.

Conductivity Analyses. In addition to the thermal properties mentioned above, we were interested in exploring the conductivity behavior of these polymer/ salt blend systems because of our previous research on solid polymer electrolytes. ${ }^{30,31}$ Figure 3 displays the conductivities of the zinc salt/P2VP and zinc salt/P4VP blends having varying zinc salt contents. Three distinct stages are observed in Figure 3 . In the first stage, at low zinc content, the conductivity increases upon increasing the amount of the salt since the zinc perchlorate is totally dissociated. In the second stage, the conductivity levels off, or slightly decreases, with increasing zinc salt content. We interpret this phenomenon to be a result of an increase in the zinc perchlorate ion-dipole interaction; the decreased mobility of the cations offsets the increased number of cations. In the third stage, after totally coordination at ca. 40 wt \% zinc salt, the conductivity increases with addition of more zinc salt.

Analyses by Infrared Spectroscopy. Analysis of infrared spectra shows that the shifts are observed in the absorption peaks of the polymer structures that are caused by specific ion-dipole interactions in these systems. According to previous reports, the characteristic absorption peaks of P2VP structures exist at 1590, $1473,1433,993$, and $625 \mathrm{~cm}^{-1}$ for the pyridine ring stretching mode and $733 \mathrm{~cm}^{-1}$ for the $\mathrm{C}-\mathrm{H}$ pyridine bending mode, and those for P4VP exist at 1597, 1557, 1050, 993, and $627 \mathrm{~cm}^{-1}$. In addition, the absorption peaks of pure zinc perchlorate appear at 600-650 and $1050-1200 \mathrm{~cm}^{-1}$ (not shown in this study). Within this spectrum, the absorptions at 627 and $635 \mathrm{~cm}^{-1}$ are caused by $v\left(\mathrm{ClO}_{4}^{-}\right)$in the zinc salt, corresponding to the free and contact ions, respectively. ${ }^{30}$ U nfortunately, the stretching peak of poly(vinylpyridine)s also appears in this region, at $625 \mathrm{~cm}^{-1}$, so it becomes too complicated to quantitatively analyze the degree of interaction of the zinc salt and the polymer using these peaks as probes.

For convenience, we turned our attention to the quantitative analyses using other infrared absorption peaks as probes, with and without the addition of the zinc salt. Figure 4 displays scale-expanded infrared spectra (1550 and $1630 \mathrm{~cm}^{-1}$ ) of the zinc salt/P2VP and zinc salt/P4VP blends in the region of the pyridine ring modes. We concentrated on the unperturbed bands at 1590 and $1597 \mathrm{~cm}^{-1}$ for P2VP and P4VP, respectively, 


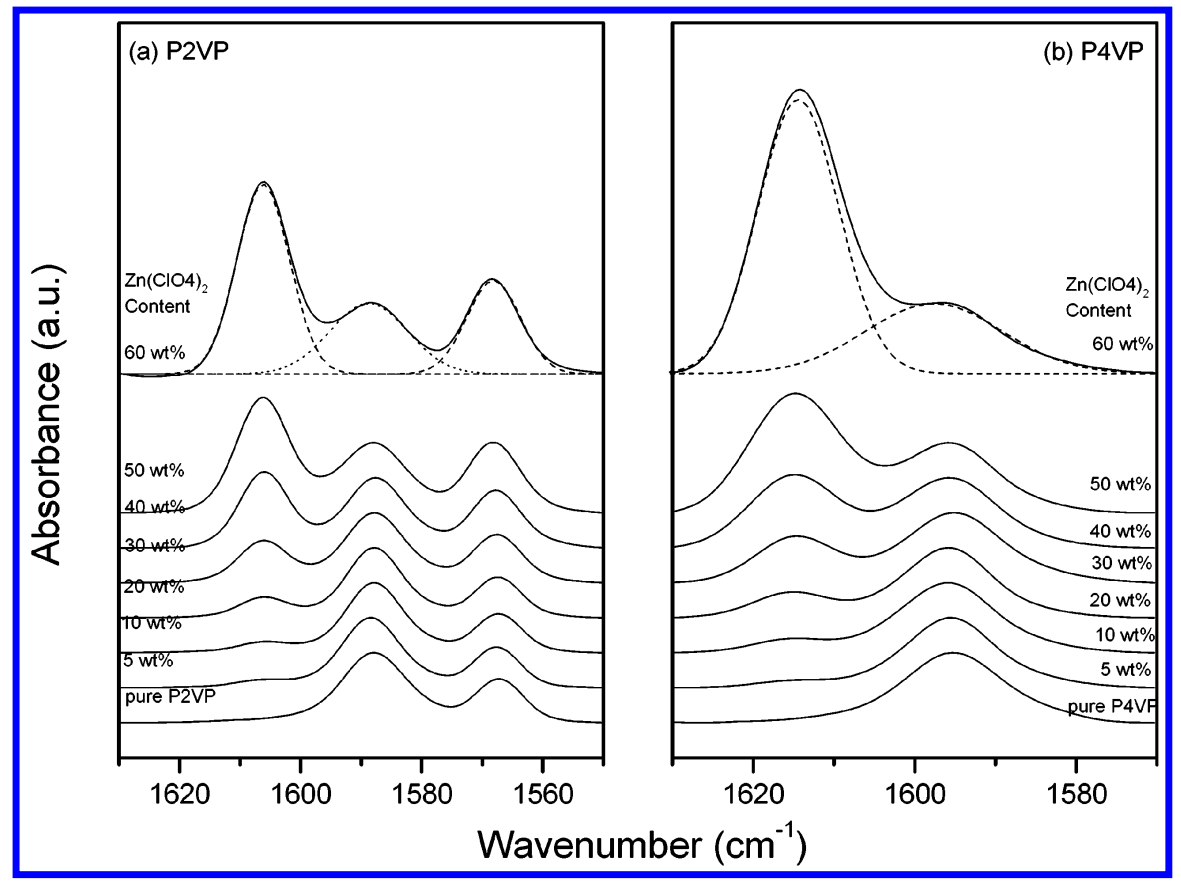

Figure 4. FTIR spectra recorded at $120^{\circ} \mathrm{C}$ in the region $1630-1550 \mathrm{~cm}^{-1}$ for the (a) zinc salt/P2VP and (b) zinc salt/P4VP blends.

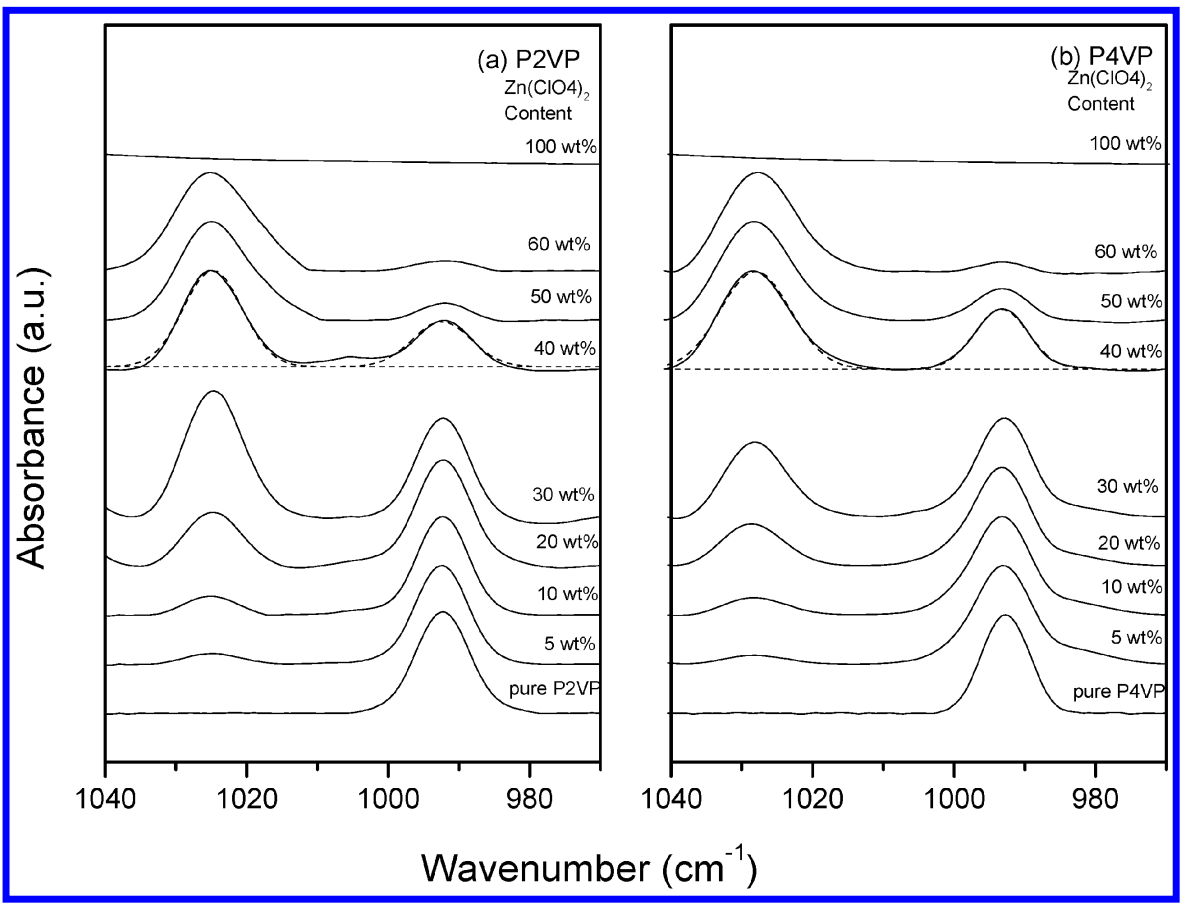

Figure 5. FTIR spectra recorded at $120^{\circ} \mathrm{C}$ in the region $1040-970 \mathrm{~cm}^{-1}$ for the (a) zinc salt/P2VP and (b) zinc salt/P4VP blends.

since these absorptions do not overlap with those of zinc perchlorate. Upon addition of $\mathrm{Zn}\left(\mathrm{ClO}_{4}\right)_{2}$, new bands appear at $1605 \mathrm{~cm}^{-1}$ (P2VP) and $1615 \mathrm{~cm}^{-1}$ (P4VP), which we assign as being due to the zinc cation coordinating with pyridine rings. These results are consistent with those previously reported by Pires et al., ${ }^{8}$ who identified the pyridine ring in P4VP as a $\pi$-bonding ligand when coordinated to the cation. Therefore, the higher energy of this new absorption is the result of the formation of such a coordination complex.

When compared with the poly(hydroxyl methacrylate)/poly(vinyl pyridine)s hydrogen-bonding blend systems, ${ }^{33}$ the significantly larger differences observed here in the position of the absorption peaks of the free and metal-complexed systems $\left(15-18 \mathrm{~cm}^{-1}\right)$ relative to those in the hydrogen-bonding system $\left(3-5 \mathrm{~cm}^{-1}\right)$, which suggests that stronger interactions exist in this metalcomplexed system. Figure 5 shows that a similar phenomenon exists with respect to the characteristic band at $993 \mathrm{~cm}^{-1}$ for P2VP and P4VP. U pon addition of $\mathrm{Zn}\left(\mathrm{ClO}_{4}\right)_{2}$, new bands appear at $1025 \mathrm{~cm}^{-1}$ for the P2VP blends and at $1028 \mathrm{~cm}^{-1}$ for the P4VP blends. The difference in the positions of the peak absorption between free and coordinated pyridine units of the P4VP blend is ca. $3 \mathrm{~cm}^{-1}$ larger than that of the P2VP blend, which indicates again that the interactions in the zinc salt/P4VP blend are greater than those in the zinc salt/ P2VP blend. All these pyridine frequencies are clearly split into two bands (free and coordinated), which can be fitted well to the Gaussian function. For brevity, the 


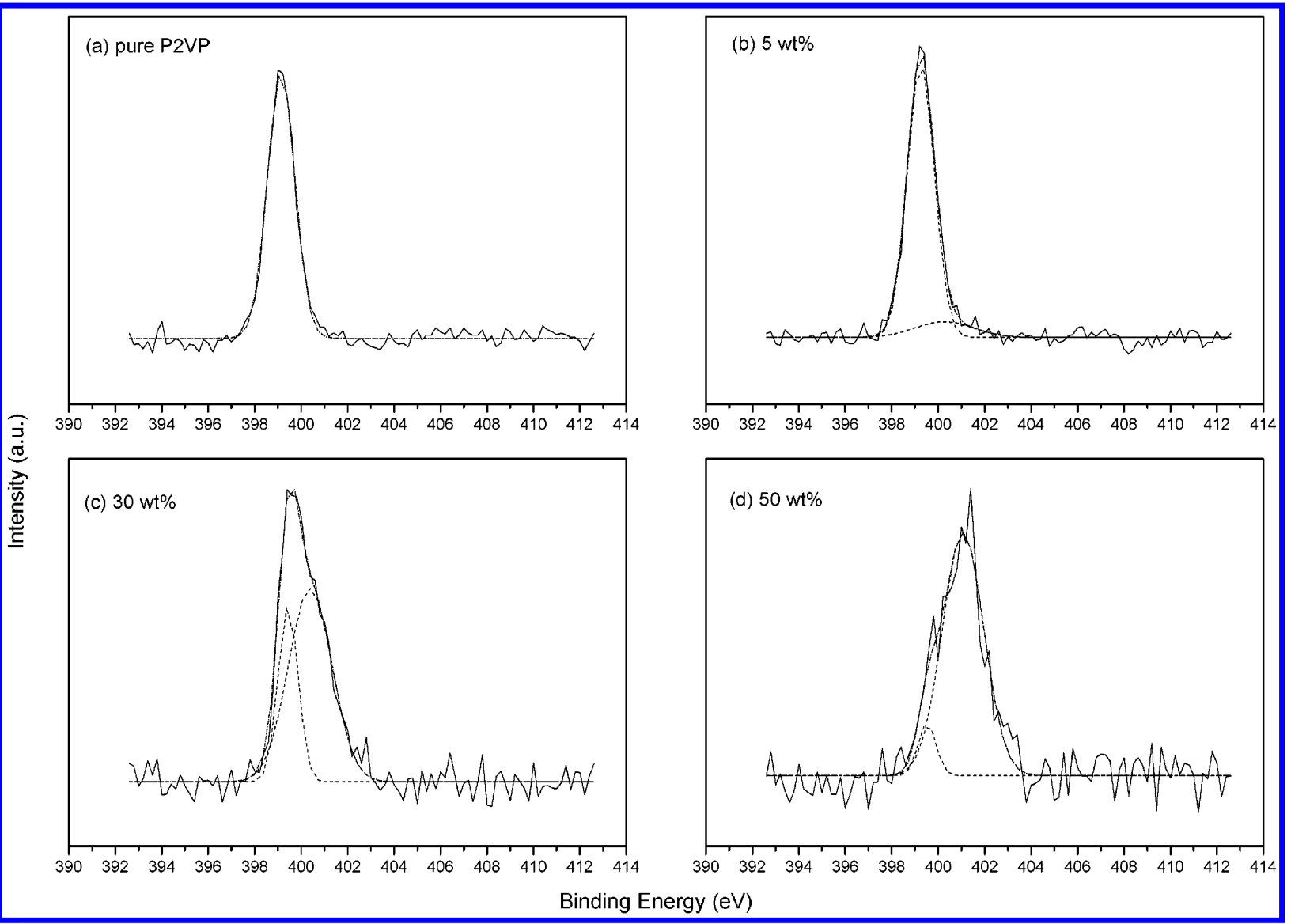

Figure 6. $\mathrm{N}_{1 \mathrm{~s}}$ spectra of zinc salt/P2VP blends having varying zinc salt content.

Table 2. Results of Curve Fitting of the Area Fraction of Pyridine Units Involved in Ion-Dipole Interaction in the Zinc Salt/P2VP and Zinc Salt/P4VP Blends at $120^{\circ} \mathrm{C}$

\begin{tabular}{|c|c|c|c|c|c|c|}
\hline \multirow[b]{2}{*}{ wt \% } & \multicolumn{3}{|c|}{ free pyridine ring } & \multicolumn{3}{|c|}{ coordination pyridine ring } \\
\hline & $v, \mathrm{~cm}^{-1}$ & $\mathrm{~W}_{1 / 2}, \mathrm{~cm}^{-1}$ & $A_{f}, \%$ & $v, \mathrm{~cm}^{-1}$ & $\mathrm{~W}_{1 / 2}, \mathrm{~cm}^{-1}$ & $A_{i}, \%$ \\
\hline \multicolumn{7}{|c|}{ Zn Salt/P2VP } \\
\hline $0 / 100$ & 1590 & 12 & 100. 0 & & & \\
\hline $10 / 90$ & 1588 & 10 & 90.4 & 1606 & 10 & 9.6 \\
\hline $20 / 80$ & 1588 & 10 & 78.9 & 1606 & 9 & 21.1 \\
\hline $40 / 60$ & 1588 & 13 & 53.3 & 1606 & 9 & 46.7 \\
\hline $60 / 40$ & 1589 & 13 & 35.2 & 1606 & 9 & 64.8 \\
\hline \multicolumn{7}{|c|}{ Zn Salt/P4VP } \\
\hline $0 / 100$ & 1595 & 14 & 100. 0 & & & \\
\hline $10 / 90$ & 1596 & 13 & 86.7 & 1615 & 10 & 13.3 \\
\hline $20 / 80$ & 1596 & 12 & 76.0 & 1615 & 10 & 24.0 \\
\hline $40 / 60$ & 1596 & 13 & 52.9 & 1615 & 11 & 47.1 \\
\hline $60 / 40$ & 1598 & 17 & 29.6 & 1615 & 10 & 70.4 \\
\hline
\end{tabular}

selected results of the subsequent curve fitting are summarized in Tables 2 and 3, which indicate that the area fraction for the coordination interaction of the pyridine ring increases with increasing zinc perchlorate content, and the area fraction of the pyridine groups undergoing coordination interactions in the P4VP blend is higher than that in the P2VP blends over the entire range of compositions.

Analyses by X-ray Photoelectron Spectroscopy. Figures 6 and 7 show the $N$ 1s spectra of the zinc salt/ P2VP and zinc salt/P4VP blends, respectively. The N 1s spectra of both pure P2VP and pure P4VP show a symmetric peak at ca. $399.1 \mathrm{eV}$. The $\mathrm{N}$ 1s peaks from the blends are asymmetric and broader, however, and each peak can be split into two component peaks. One peak remains at ca. $399.1 \mathrm{eV}$, and the other appears at ca. $400.5 \mathrm{eV}$. The occurrence of the new peak having
Table 3. Results of Curve Fitting of the Area Fraction of Pyridine Units Involved in Ion-Dipole Interaction in the Zinc Salt/P2VP and Zinc Salt/P4VP Blends at $120{ }^{\circ} \mathrm{C}$

\begin{tabular}{|c|c|c|c|c|c|c|}
\hline \multirow[b]{2}{*}{ wt \% } & \multicolumn{3}{|c|}{ free pyridine ring } & \multicolumn{3}{|c|}{ coordination pyridine ring } \\
\hline & $v, \mathrm{~cm}^{-1}$ & $\mathrm{~W}_{1 / 2}, \mathrm{~cm}^{-1}$ & $A_{f}, \%$ & $v, \mathrm{~cm}^{-1}$ & $\mathrm{~W}_{1 / 2}, \mathrm{~cm}^{-1}$ & $A_{i}, \%$ \\
\hline \multicolumn{7}{|c|}{ Zn Salt/P2VP } \\
\hline 0/100 & 993 & 8 & 100. 0 & & & \\
\hline $10 / 90$ & 993 & 7 & 85.7 & 1025 & 7 & 14.3 \\
\hline $20 / 80$ & 993 & 7 & 70.2 & 1025 & 7 & 29.8 \\
\hline $40 / 60$ & 993 & 5 & 32.0 & 1025 & 7 & 68.0 \\
\hline $60 / 40$ & 993 & 7 & 7.6 & 1025 & 11 & 92.4 \\
\hline \multicolumn{7}{|c|}{ Zn Salt/P4VP } \\
\hline 0/100 & 993 & 7 & 100. 0 & & & \\
\hline $10 / 90$ & 993 & 9 & 83.2 & 1028 & 8 & 16.8 \\
\hline $20 / 80$ & 993 & 9 & 67.7 & 1028 & 8 & 32.3 \\
\hline $40 / 60$ & 993 & 8 & 17.0 & 1028 & 10 & 83.0 \\
\hline $60 / 40$ & 993 & 5 & 6.6 & 1028 & 10 & 93.4 \\
\hline
\end{tabular}

higher binding energy indicates that a fraction of the pyridine nitrogen atom interacts with zinc cations. A similar result has been observed in the poly(styrenesulfonate) zinc salt/P4VP system. ${ }^{20}$ This result also shows that the ionic interaction is greater than that observed in the hydrogen-bonding PVPh/P4VP complex system $(400 \mathrm{eV}) .{ }^{34}$ The results from curve fitting are summarized in Table 4, where the area fraction of the pyridine rings involved in ion-dipole interactions increases with increasing zinc perchlorate content. The area fraction of the pyridine groups in the P4VP blend that are involved in ion-dipole interactions is greater than the for the P2VP blends over the entire range of compositions.

Analyses by Solid-State NMR Spectroscopy. Solidstate NMR spectroscopy provides further insight into the phase behavior and morphology of the polymer 


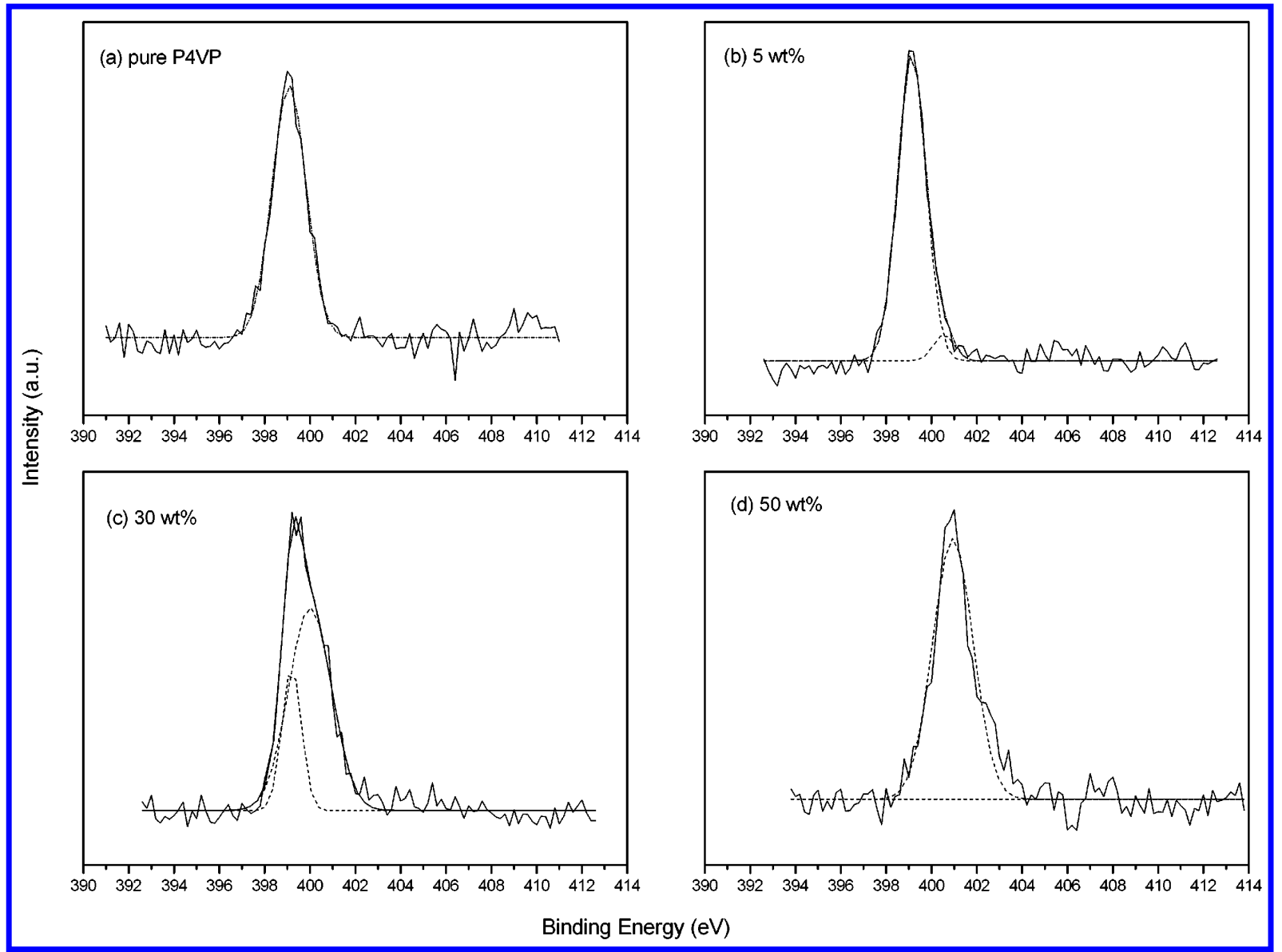

Figure 7. $\mathrm{N}_{1 \mathrm{~s}}$ spectra of zinc salt/P4VP blend having varying zinc salt content.

Table 4. Results of Curve Fitting of the Area Fraction of Pyridine Units Involved in Ion-Dipole Interaction in the Zinc Salt/P2VP and Zinc Salt/P4VP Blends at Room Temperature

\begin{tabular}{|c|c|c|c|c|c|c|}
\hline \multirow[b]{2}{*}{ wt \% } & \multicolumn{3}{|c|}{ free pyridine ring } & \multicolumn{3}{|c|}{ coordination pyridine ring } \\
\hline & $\mathrm{BE}, \mathrm{cm}^{-1}$ & $\mathrm{~W}_{1 / 2}, \mathrm{~cm}^{-1}$ & $A_{f}, \%$ & $\mathrm{BE}, \mathrm{cm}^{-1}$ & $\mathrm{~W}_{1 / 2}, \mathrm{~cm}^{-1}$ & $A_{i}, \%$ \\
\hline \multicolumn{7}{|c|}{ Zn Salt/P2VP } \\
\hline $0 / 100$ & 399.1 & 1.2 & 100 & & & \\
\hline $5 / 95$ & 399.4 & 0.9 & 94.1 & 400.4 & 1.4 & \\
\hline $30 / 70$ & 399.5 & 0.8 & 29.6 & 401 & 1. & \\
\hline $50 / 50$ & 399.3 & 1.2 & 8.5 & 400.3 & 2.5 & \\
\hline \multicolumn{7}{|c|}{ Zn Salt/P4VP } \\
\hline $0 / 100$ & 399.1 & 1.5 & 100 & & & \\
\hline 5/95 & 399.1 & 1.2 & 88.2 & 400.6 & 0.9 & \\
\hline $30 / 70$ & 399.2 & 0.9 & 24.3 & 400.0 & 1.9 & 15 \\
\hline $50 / 50$ & & & & 401.0 & 1.8 & 100 \\
\hline
\end{tabular}

blends involved in coordination to the ion. Figure 8

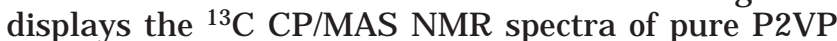
and pure P4VP and their blends with zinc perchlorate. Pure P2VP has peaks for six major resonances, and pure P4VP has five. Chart 1 indicates the atom numbering used for the peak assignments in Figure 8. Table 5 summarizes the values of the chemical shifts observed in the ${ }^{13} \mathrm{C} C P / M A S ~ N M R$ spectra of the zinc salt/P2VP and zinc salt/P4VP blends. Compared with the ${ }^{13} \mathrm{C} \mathrm{CP/}$ MAS NMR spectra of the pure polymers, the spectra of the zinc salt/P2VP and zinc salt/P4VP blends display significant changes, especially for the resonances of the carbon atoms involved in intermolecular ion-dipole interactions. For example, peaks of the pyridine carbon atoms (C-5 and C-6) of pure P2VP, positioned at 149.8 and $165.0 \mathrm{ppm}$, and that for C-11 of pure P4VP show upfield shifts of 1.4, 1.8, and 0.9 ppm, respectively, in
Chart 1. Chemical Structures of P2VP and P4VP and Their Atom Numbering Schemes

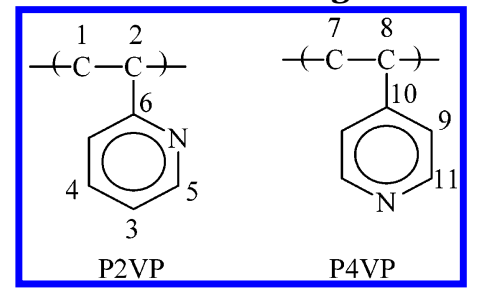

the presence of 50 wt \% zinc salt, relative to those of their polymers. Furthermore, the peaks of the methylene carbon atom of P2VP and P4VP (C-1 and C-7, respectively) also show upfield shifts upon increasing the content of zinc salt, and a new peak at $32.5 \mathrm{ppm}$ appears in these two blend systems. The peaks for the C-2, C-3, and C-4 atoms of pure P2VP and C-8 and C-9 of pure P4VP show downfield shifts, however, with increasing zinc content. In addition, upon increasing the zinc salt content, new shoulders appear at ca. 143.5 ppm for the signal of the C-4 atom and at ca. 126.3 ppm for C-9. All these shifts relate to changes in bond angles and intermolecular distances to the atoms' nearest neighbors.

Analyses by Wide-Angle X-ray Diffraction (WAXD). The morphologies of the zinc salt/P2VP and zinc salt/P4VP blends were studied by WAXD to characterize the microstructures and d spacings of these blends with respect to their zinc contents. Figure 9 shows the WAXD patterns of various zinc salt/P2VP and zinc salt/P4VP blend ratios. Both P2VP and P4VP are amorphous polymers and provide two broad amorphous peaks, and the crystalline diffraction pattern of zinc 


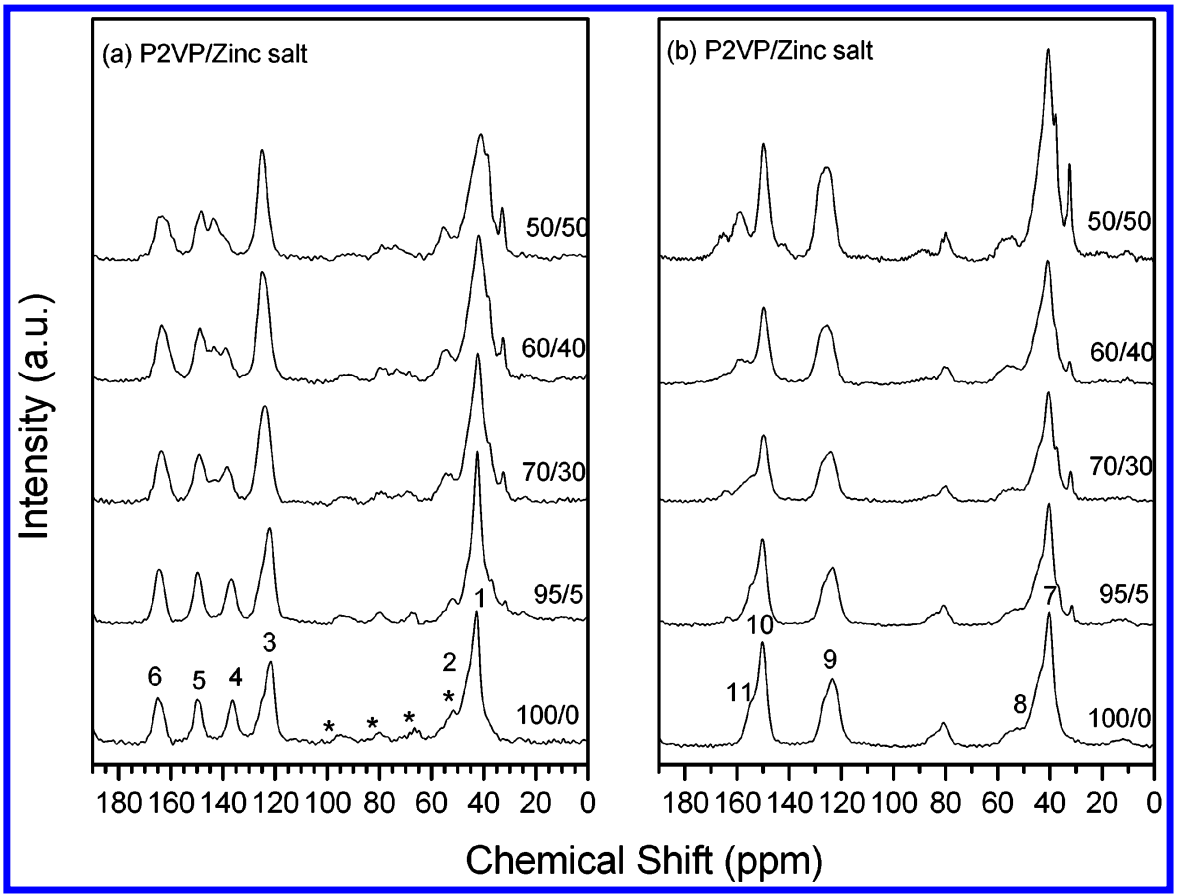

Figure 8. ${ }^{13} \mathrm{C}$ CPMAS NMR spectra recorded at room temperature of the (a) zinc salt/P2VP and (b) zinc salt/P4VP blends.

Table 5. Chemical Shift (ppm) Observed in the ${ }^{13} \mathrm{C}$ CP/MAS/DD NMR Spectra of P2VP and P4VP and of Their Zinc Salt Blends

\begin{tabular}{clccccc}
\hline \multirow{2}{*}{$\begin{array}{c}\text { Zn salt } \\
\text { wt \% }\end{array}$} & \multicolumn{7}{c}{ C-1 } & C-2 & C-3 & C-4 & C-5 & C-6 \\
\cline { 2 - 7 } & 42.7 & 51.8 & 121.7 & 136.3 & 149.8 & 165.0 \\
5 & 42.4 & 52.0 & 122.1 & 136.8 & 149.6 & 164.6 \\
30 & $42.2(32.5)$ & 52.8 & 123.9 & $138.5(143.3)$ & 149.0 & 163.8 \\
40 & $41.8(32.5)$ & 54.2 & 124.9 & $138.9(143.6)$ & 148.8 & 163.1 \\
50 & $41.0(32.9)$ & 55.4 & 124.9 & $139.2(143.8)$ & 148.4 & 163.2 \\
\hline \multirow{2}{*}{ Zn salt } & \multicolumn{7}{c}{ P4VP } \\
wt \% & C-7 & C-8 & C-9 & C-10 & C-11 \\
\hline 0 & 40.2 & & 52.6 & 123.5 & 150.2 & 154.5 \\
5 & 40.4 & & 53.4 & 123.7 & 150.2 & 154.4 \\
30 & 40.6 & 54.4 & 124.1 & 149.8 & 154.1 \\
40 & $40.8(32.5)$ & 56.2 & 125.5 & 149.8 & 153.7 \\
50 & $40.8(32.5)$ & 56.8 & 126.3 & 149.8 & 153.6
\end{tabular}

perchlorate is observed. In previous studies, 35,36 two amorphous peaks were also observed for polystyrene containing pendant groups. The first peak, at a lower diffraction angle, is due to the intermolecular distance between the polymer chains; the second peak, at higher diffraction angle, arises from the inter-phenyl group distance. The chemical structures of poly(vinylpyridine)s are similar to that of polystyrene, and they also possess pendant groups. Therefore, two amorphous peaks are also expected for these poly(vinylpyridine)s in their WAXD patterns. The $d$ spacing can be estimated from these two amorphous peaks by using the Bragg relation. For pure P2VP and pure P4VP, the interchain distances are 7.8 and $8.6 \AA$, respectively, while the distances between pendant groups are 4.4 and $4.0 \AA$, respectively. We observed a decrease in the distance between the pedant groups with increasing zinc salt content, which is because the zinc cation coordination results in transient cross-links between pendant pyridine groups of the polymer chains. ${ }^{37}$ The observed decrease in the interchain distance at a zinc salt content bel ow $10 \mathrm{wt} \%$ is explained by the fact that the strength of the cross-links increases and causes interchain contraction. The interchain distance for the zinc salt/poly(vinylpyridine) blends increases as the concentration of the zinc salt rises above $10 \mathrm{wt} \%$. This result is explained by the fact that the $\mathrm{ClO}_{4}{ }^{-}$anions are weakly solvated and distributed among the polymer chains. These large solvated $\mathrm{ClO}_{4}^{-}$anions cause chain expansion, as the polymer chains are forced apart; a similar result has been observed also for a $\mathrm{LiClO}_{4} / \mathrm{PPO}$ complex. ${ }^{38}$

Proton Spin-Lattice Relaxation Time in the Rotating Frame Analyses. We examined the spinlattice relaxation times in the rotating frame $\left(\mathrm{T}_{1_{\rho}}{ }^{\mathrm{H}}\right)$ to measure the homogeneity of the zinc salt/poly(vinylpyridine)s blends on the molecular scale. Table 6 summarizes values of $\mathrm{T}_{1 \rho}{ }^{\mathrm{H}}$ derived from the binary exponential analysis. The blend having low zinc salt content exhibits a single exponential, and the value of $\mathrm{T}_{1 \rho}{ }^{\mathrm{H}}$ decreases upon increasing the zinc content. This observation indicates that the domain size and interchain distance of the zinc salt/poly(vinylpyridine)s blends decrease as a result of the strong ion-dipole interactions between the zinc cations and the pyridine rings, which is consistent with the earlier analysis by WAXD at lower zinc salt content. The biexponential decay gradually appears, however, at higher zinc salt contents. The maximum diffusive path length, $L$, can be estimated using the approximate equation ${ }^{39-41}$

$$
\mathrm{L}=(6 \mathrm{DT})^{1 / 2}
$$

For a value of $\mathrm{T}_{1 \rho}{ }^{\mathrm{H}}$ of $5 \mathrm{~ms}$ and an effective spin diffusion coefficient, D, of $10^{-16} \mathrm{~m}^{2} \mathrm{~s}^{-1}$, the micro-phase-separation dimensions of these zinc salt/poly(vinylpyridine) blends at higher zinc salt are higher than $2 \mathrm{~nm}$ in the amorphous phase. This result is consistent with our earlier WAXD analysis, which suggested that the interchain distance or domain size increases, and micro phase separation occurs, at higher zinc salt content. Therefore, as Figure 2 shows, the low molecular weight inorganic salt acts as a plasticizer to decrease the gl ass transition temperature of zinc salt/poly(vinylpyridine) blends at higher zinc salt content. Furthermore, the zinc salt/P4VP blend shows the biexponential decay at 30 

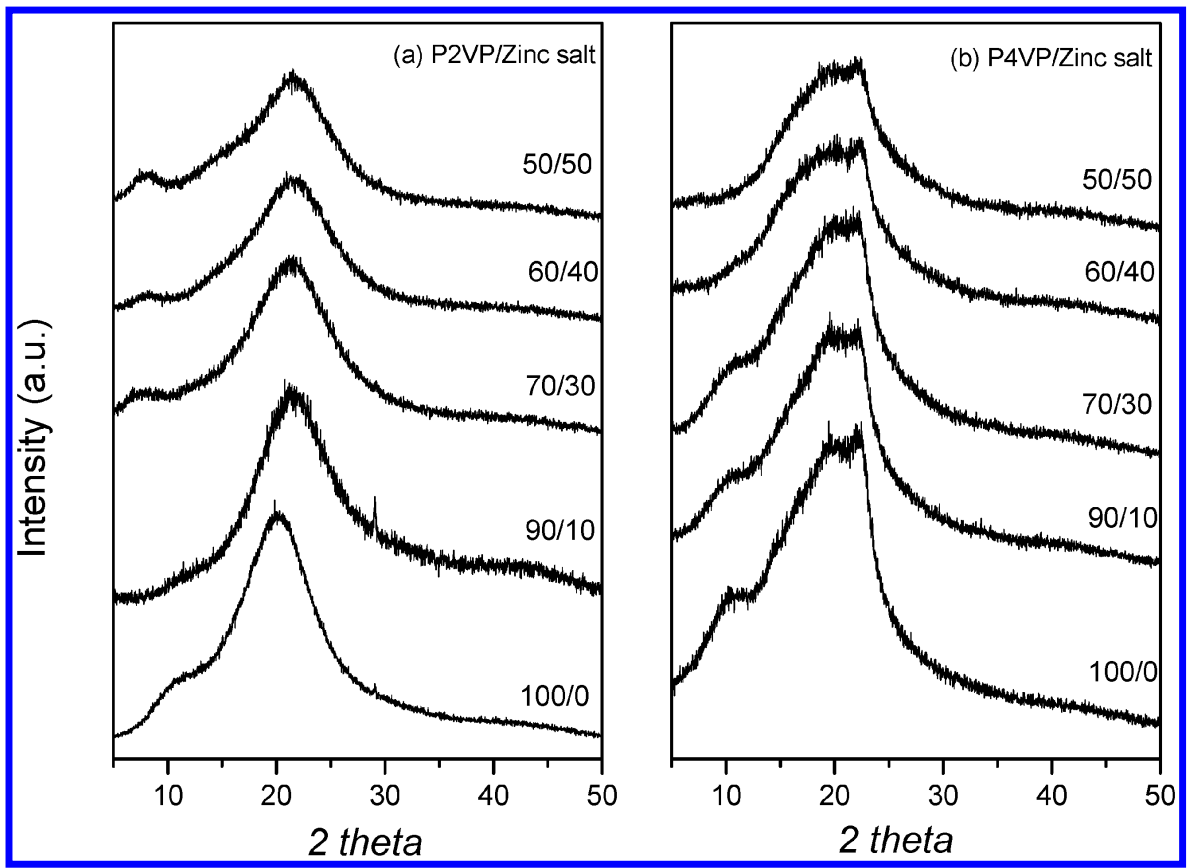

Figure 9. Wide-angle X-ray diffraction analyses of the (a) zinc salt/P2VP and (b) zinc salt/P4VP blends.

Table 6. Values of $\mathrm{T}_{1 \rho}{ }^{\mathrm{H}}$ of Pure P2VP and Pure P4VP and of the Various Zinc Salt/P2VP and Zinc Salt/P4VP Blends at Room Temperature

\begin{tabular}{|c|c|c|c|c|c|}
\hline \multirow{2}{*}{$\begin{array}{c}\text { Zn salt } \\
\text { wt \% }\end{array}$} & \multicolumn{3}{|c|}{ P2VP } & \multicolumn{2}{|c|}{ P4VP } \\
\hline & $C-3$ & C-5 & $C-6$ & $\mathrm{C}-3$ & C-4 \\
\hline 0 & 7.16 & 6.61 & 6.67 & 8.36 & 8.79 \\
\hline 5 & 6.12 & 6.19 & 6.00 & 6.59 & 6.89 \\
\hline 30 & 6.13 & 6.21 & $5.95 / 8.39$ & $5.31 / 8.63$ & $5.15 / 6.86$ \\
\hline 50 & $5.67 / 7.59$ & $4.91 / 6.58$ & $4.46 / 8.45$ & $5.07 / 6.72$ & $4.88 / 6.87$ \\
\hline
\end{tabular}

wt \%, but the zinc salt/P2VP blend requires 40 wt \% zinc salt. This result also implies that the ionic interaction of the zinc salt/P4VP blend is greater than that of the zinc salt/P2VP blend since the zinc salt/P4VP blend is saturated with a lower zinc salt content.

\section{Conclusions}

The position of the nitrogen atom of the pyridine rings in these polymers is a characteristic that influences their coordination properties. The data obtained from FTIR, XPS, and solid-state NMR spectroscopies show that P4VP has a greater ability than does P2VP to interact with the zinc salt because of the effect of steric hindrance at the nitrogen atoms in P2VP. The glass transition temperatures of the P2VP and P4VP are increased by 119 and $154^{\circ} \mathrm{C}$, relative to those of their pure polymers upon blending with the zinc perchlorate at 40 and 30 wt \%, respectively. On the basis of the extended configuration entropy model equation, the strength of the interaction of the zinc salt/P4VP blend (3.60) is greater than that of the zinc salt/P2VP blend (3.55). Furthermore, the special diffraction pattern observed in wide-angleX-ray diffraction analysis at high salt concentration indicates that the micro phase separation occurs, which is consistent with the two relaxation times observed in solid-state NMR spectroscopy.

Acknowledgment. We thank the National Science Council, Taiwan, Republic of China, for financially supporting this research under Contract No. NSC-922216-E-009-018.

\section{References and Notes}

(1) Hong, S. U.; J in, J . H.; Won, J .; Kang, Y. S. Adv. Mater. 2000, $12,968$.

(2) Kim, J. H.; Min, B. R.; Kim, C. K.; Won, J .; Kang, Y. S. Macromolecules 2001, 34, 6.

(3) Kim, J. H.; Min, B. R.; Won, J .; Kang, Y. S. Macromolecules 2003, 36, 4577.

(4) Balfiore, L. A.; McCurdie, M. P.; U eda, E. Macromolecules 1993, 26, 6908.

(5) Kuo, S. W.; Lin, C. L.; Chang, F. C. Polymer 2002, 43, 3943.

(6) Rodrigues, J . R. S.; Gonçalves, D.; Mangrich, A. S.; Soldi, V.; Bertolino, J. R.; Pires A. T. N. Adv. Polym. Technol. 2000, 19, 113.

(7) Agnew, N. H. L. Polym. Sci., Polym. Chem. Ed. 1976, 14, 2819.

(8) Pires, A. T. N.; Cheng, C.; Belfiore, L. A. ACS Proc. Div. Polym. Mater. Sci. Eng. 1989, 61, 466.

(9) Wang Y. M.S. Thesis, Colorado State University, 1991.

(10) Belfiore, L. A.; Pires, A. T. N.; Wang, Y.; Graham, H.; Ueda, E. Macromolecules 1992, 25, 1411.

(11) Allan, J . R.; Carson, B. R.; Turvey, K.; Birnie, J .; Gerrard, D. L. Eur. Polym. L. 1991, 27, 665.

(12) Allan, J. R.; Carson, B. R.; Turvey, K.; Birnie, J .; Gerrard, D. L. Thermochim. Acta 1991, 180, 38.

(13) Belfiore, L. A. Polym. Prepr. (Am. Chem. Soc. Div. Polym. Chem.) 1992, 33, 925.

(14) Belfiore, L. A.; Graham, H.; U eda, E. Macromolecules 1992, $25,2935$.

(15) Pearson R. G. In Survey of Progress in Chemistry; Scott, Ed.; Academic Press: New York, 1969; Vol. 6, Chapter 1.

(16) Coleman, M. M.; Graf, J . F.; Painter, P. C. Specific Interactions and the Miscibility of Polymer Blends; Technomic Publishing: Lancaster, PA, 1991.

(17) Kuo, S. W.; Chang, F. C. Macromolecules 2001, 34, 5224.

(18) Kuo, S. W.; Chang, F. C. Macromolecules 2001, 34, 7737.

(19) Goh, S. H.; Lee, S. Y.; Zhou, X.; Tan, K. L. Macromolecules 1999, 32, 942

(20) Goh, S. H.; Lee, S. Y.; Zhou, X.; Tan, K. L. Macromolecules 1998, 31, 4260.

(21) J iao, H.; Goh, S. H.; Valiyaveettil, S. Macromolecules 2001, 34, 7162.

(22) Hill, D. J . T.; Whittaker, A. K.; Wong, K. W. Macromolecules 1999, 32, 5285.

(23) Zhang, X.; Takegoshi, K.; Hikichi, K. Macromolecules 1991, 24, 5756.

(24) Goh, S. H.; Yi, J . Z. Polymer 2001, 42, 9316.

(25) Mi, Y.; Zheng, S. Polymer 2003, 44, 1067.

(26) Kuo, S. W.; Chang, F. C. Macromolecules 2001, 34, 4089.

(27) Eisenberg, A.; Rinaudo, M. Polym. Bull. 1990, 24, 671.

(28) Kim, J. H.; Min, B. R.; Won, J.; Kang, Y. S. L. Phys. Chem. B 2003, 107, 5901. 
(29) Kim, J . H.; Min, B. R.; Kim, C. K.; Won, J .; Kang, Y. S. Macromolecules 2002, 35, 5250.

(30) Chen, S. W.; Wu, H. D.; Chang, F. C. Polymer 2002, 43, 5011.

(31) Chen, S. W.; Chang, F. C. Polymer 2002, 43, 5281.

(32) Lee, J . Y.; Painter, P. C.; Coleman, M. M. Macromolecules 1988, 21, 954.

(33) Cesteros, L. C.; I sasi, J . R.; Katime, I. Macromolecules 1993, $26,7256$.

(34) Jiao, H.; Goh, S. H.; Valiyaveettil, S. Macromolecules 2001 34, 7162.

(35) Mitchell, G. R.; Windle, A. H. Polymer 1984, 25, 906.

(36) Aguilar-Vega, M.; Paul, D. R. L. Polym. Sci.,Part B: Polym. Phys. 1993, 31, 1577.
(37) Choi, S.; Kim, J. K.; Kang, Y. S. Macromolecules 2001, 34, 9087.

(38) Carlsson, P.; Mattsson, B.; Swenson, J .; Borjesson, L.; Torell, L. M.; McGreevy, R. L.; Howell, W. S. Electrochim. Acta 1998 43, 1545.

(39) McBrierty, V. J .; Douglass, D. C. L. Polym. Sci., Macromol. Rev. 1981, 16, 295.

(40) Demco, D. E.; J ohansson, A.; Tegenfel dt, J . Solid State Nucl. Maqn. Reson. 1995, 4, 13

(41) Clauss J .; Schmidt-Rohr K, W. H. Acta Polym. 1993, 44, 1.

(42) Van-Krevelen, D. W. Properties of Polymer; Elsevier: Amsterdam, 1990.

MA035655+ 